\title{
Structure of a Lipid-bound Viral Membrane Assembly Protein Reveals a Novel Modality for Interacting with Lipid Bilayer
}

Prabhat Pathak+1, Shuxia Peng+1, Xiangzhi Meng+2, Yue Hanı, Bing Zhang1, Fushun

Zhang2, Yan Xiang*2, Junpeng Deng*1

IDepartment of Biochemistry and Molecular biology, Oklahoma State University, 246

Noble Research Center, Stillwater, OK 74078, USA.

2Department of Microbiology, Immunology and Molecular Genetics, University of Texas

Health Science Center at San Antonio, 7703 Floyd Curl Drive, San Antonio, TX 78229 ,

USA

\begin{abstract}
Cellular membranes are maintained as closed compartments, only broken up transiently during membrane reorganization or lipid transportation. However, open-ended membranes, likely derived from scissions of the endoplasmic reticulum (ER), persist in vaccinia virus-infected cells during the assembly of the viral envelope. A group of viral membrane assembly proteins (VMAPs) were identified as essential for this process. Here, we report the crystal structure of the largest VMAP, named A6, which is a soluble protein with two alpha-helical domains. The larger $\mathrm{C}$-terminal domain forms a unique cage that encloses multiple glycerophospholipids with a lipid bilayer-like configuration. The smaller N-terminal domain does not bind lipid but negatively affects lipid-binding by A6. Mutations of key hydrophobic residues lining the lipid-binding cage disrupt lipid-binding and abolish viral replication. Our results reveal a novel protein modality for interacting with lipid-bilayer and provide the molecular insight on a viral machinery involved in generating and/or stabilizing open-ended membranes.
\end{abstract}

